\title{
A PERSPECTIVA DA VULNERABILIDADE NA AVALIAÇÃO DO PÉ DIABÉTICO SOB A ÓTICA DE ENFERMEIROS*
}

\author{
Leandra de Fátima Bento ${ }^{1}$, Agnelo Denis Vieira ${ }^{2}$, Leandro Cleverson Chaves ${ }^{3}$, Marcia Regina Cubas ${ }^{4}$
}

\begin{abstract}
RESUMO: O objetivo deste estudo foi descrever as condições determinantes sociais em saúde, identificadas por enfermeiros como relevantes para o estabelecimento de vulnerabilidades da pessoa com Diabetes Mellitus. Pesquisa exploratória descritiva cuja metodologia foi dividida em duas fases: identificação das condições determinantes sociais em saúde capazes de influenciar no desenvolvimento do pé diabético; e avaliação das condições determinantes sociais em saúde por enfermeiros. A pesquisa foi realizada entre os meses de agosto e novembro de 2014, com enfermeiros atuantes no município de Curitiba e Região Metropolitana. Das 68 condições determinantes sociais em saúde, 20 foram consideradas influentes para o desenvolvimento do pé diabético. Destas, apenas 3 pertencem à vulnerabilidade social. Conclui-se que a dimensão social da vulnerabilidade ainda é fracamente reconhecida pelos enfermeiros como capaz de influenciar no desenvolvimento do pé diabético. DESCRITORES: Determinantes sociais de saúde; Vulnerabilidade em saúde; Pé diabético.
\end{abstract}

\section{THE PERSPECTIVE OF VULNERABILITY IN THE DIABETIC FOOT EVALUATION FROM THE VIEWPOINT OF NURSES}

ABSTRACT: The aim of this study was to describe the social determinants of health, identified by nurses as relevant to the establishment of vulnerabilities of people with diabetes mellitus. A descriptive exploratory study was conducted, and its methodology was divided into two phases: identification of social determinants of health that can impact the development of diabetic foot; and evaluation of the social determinants of health by nurses. The research was conducted between August and November 2014, with nurses working in Curitiba and its metropolitan region. Of 68 social determinants of health, 20 were considered influential to the development of diabetic foot. Of these, only three belong to social vulnerability. In conclusion, the social dimension of vulnerability is still poorly recognized by nurses as capable of influencing the development of diabetic foot.

DESCRIPTORS: Social determinants of health; Health vulnerability; Diabetic foot.

\section{PERSPECTIVA DE LA VULNERABILIDAD EN LA EVALUACIÓN DEL PIE DIABÉTICO SEGÚN LA VISIÓN DE ENFERMEROS}

RESUMEN: El objetivo del estudio fue describir las condiciones sociales determinantes en salud, identificadas por enfermeros como de relevancia para el establecimiento de vulnerabilidades de la persona con Diabetes Mellitus. Investigación exploratoria con metodología dividida en dos fases: identificación de las condiciones sociales determinantes en salud capaces de influir en el desarrollo del pie diabético; y evaluación de las condiciones sociales determinantes en salud por los enfermeros. La investigación se realizó entre agosto y noviembre de 2014, con enfermeros actuantes en el municipio de Curitiba y Región Metropolitana. De las 68 condiciones sociales determinantes en salud, 20 fueron consideradas influyentes para el desarrollo del pie diabético. De ellas, solamente 3 forman parte de la vulnerabilidad social. Se concluye en que la dimensión social de la vulnerabilidad es aún francamente reconocida por los enfermeros como capaz de influir en el desarrollo del pie diabético.

DESCRIPTORES: Determinantes Sociales de la Salud; Vulnerabilidad en Salud; Pie Diabético.

\footnotetext{
*Artigo extraído da dissertação intitulada: "Os determinantes sociais de saúde no desenvolvimento do pé diabético". Pontifícia Universidade Católica do Paraná, 2015.

${ }^{1}$ Enfermeira. Mestre em Tecnologia em Saúde. Docente de Enfermagem da Universidade Tuiuti do Paraná. Curitiba, PR, Brasil. ${ }^{2}$ Engenheiro mecânico. Doutor em Engenharia Elétrica. Docente da Pontifícia Universidade Católica do Paraná. Curitiba, PR, Brasil.

${ }^{3}$ Analista de Sistemas. Centro Universitário Autônomo do Brasil. Curitiba, PR, Brasil.

${ }^{4}$ Enfermeira. Doutora em Enfermagem. Docente da Pontifícia Universidade Católica do Paraná. Curitiba, PR, Brasil.
} 


\section{- INTRODUÇÃO}

O pé diabético é considerado uma complicação incapacitante relacionada ao Diabetes Mellitus (DM) e consiste em alterações de pele, ossos e articulações dos pés que colaboram para o aparecimento de ulcerações, infecções e gangrena ${ }^{(1)}$. Seu tratamento tem alto custo, envolve hospitalizações prolongadas e nos Estados Unidos da América precede $85 \%$ das amputações em pés ${ }^{(2-4)}$. No Brasil, os internamentos relacionados a esta complicação totalizaram 12.083 internações, em 2012; 13.341 em 2013; e 13.782 em $2014^{(5)}$.

Ulcerações e amputações nos pés de indivíduos portadores de DM podem ser reduzidas de $50 \%$ a até $60 \%$ por meio de ações de prevenção e manejo da doença ${ }^{(6)}$. Tais ações são mobilizadas pela avaliação do risco a partir de protocolos direcionados à análise de aspectos biológicos do indivíduo portador de $\mathrm{DM}^{(7-11)}$.

Apesar da aplicação de protocolos de avaliação, as amputações continuam ocorrendo. Deste modo, questiona-se a efetividade de tal avaliação. Uma das hipóteses da limitação de efetividade é a incipiência de análise dos aspectos relacionados à vulnerabilidade e aos determinantes sociais em saúde (DSS). A inclusão de elementos relacionados à face social do diabético poderia ampliar a avaliação de risco, complementando-a com graus de vulnerabilidade, de modo a estabelecer intervenções mais adequadas à realidade social do indivíduo e de sua família.

O conceito de vulnerabilidade vem sendo utilizado para caracterizar grupos populacionais mais atingidos pelos aspectos sociais. A associação vulnerabilidade e adoecimento teve origem durante a epidemia da Síndrome da Imunodeficiência Humana (AIDS), pois a abordagem restrita às condições biológicas não era suficiente para explicar a disseminação do vírus ${ }^{(12)}$.

Para análise de vulnerabilidade é necessário entendê-la em três dimensões ${ }^{(12)}$ : individual, programática e social. A dimensão individual analisa a reação do indivíduo ao adoecimento ou a proteção para certas doenças. A dimensão programática abrange temas relacionados à organização dos serviços de saúde e suas respostas às necessidades de saúde das pessoas em seus contextos sociais, assim como a capacitação dos profissionais para identificar os contextos de vulnerabilidades. E a dimensão social envolve questões de grupos sociais como etnia, gênero, crenças e exclusão social; bem como a de aspectos estruturais da economia e políticas públicas ${ }^{(12)}$.

Ao associar as três dimensões da vulnerabilidade, é possível reconhecer a determinação social do processo saúde doença e se aproximar das práticas de saúde como sociais e históricas ${ }^{(13)}$.

Mundialmente, a busca pela promoção de consciência sobre a importância dos determinantes sociais e combate às iniquidades em saúde foi motivadora para proposição de comissões específicas para esta problemática, sendo o Brasil o primeiro país a criar a Comissão Nacional sobre Determinantes Sociais da Saúde (CNDSS), em 2006.

A comissão usa como referência o modelo conceitual de Dahlgren e Whitehead, no qual os DSS são estratificados em conjuntos de condições: socioeconômicas, culturais e ambientais gerais; vida e trabalho; redes sociais e comunitárias; estilo de vida dos indivíduos; e idade, gênero e fatores hereditários. Esses conjuntos representam condições que vão das mais próximas ao indivíduo até as mais distantes ${ }^{(14)}$. Dada a complexidade desta estratificação, no presente estudo, os diferentes elementos que compõem cada grupo de DSS foram denominados, isoladamente, de condição determinante social em saúde (ConDSS), de modo a diferenciar o conjunto de determinantes das condições isoladas.

No contexto da problemática apresentada, emergem duas questões: Que condições determinantes sociais em saúde têm potencial para interferir no desenvolvimento do pé diabético? Estas condições são reconhecidas por enfermeiros para que se estabeleçam graus de vulnerabilidade de modo a complementar a avaliação de riscos do pé diabético?

Neste contexto, este artigo teve como objetivo descrever as condições determinantes sociais em saúde, identificadas por enfermeiros como relevantes para o estabelecimento de vulnerabilidades da pessoa portadora de DM. 


\section{- MÉTODO}

Trata-se de uma pesquisa exploratória descritiva desenvolvida em duas fases: a identificação das ConDSS a partir da seleção dos DSS com potencial para influir no desenvolvimento do pé diabético, categorizando-as nas dimensões de vulnerabilidade individual, social e programática; e a avaliação das ConDSS, por especialistas enfermeiros.

A base empírica utilizada na primeira fase foi o relatório final da CNDSS. O critério de inclusão, adotado por um pesquisador independente, para seleção dos DSS foi a possibilidade de relação direta ou indireta com o desenvolvimento do pé diabético. Os critérios de exclusão adotados foram o relacionamento direto: ao público infantil (exemplo: baixo risco ao nascer); a grupos de doenças regionais (exemplo: morar em zona endêmica): a grupo de doenças contagiosas específicas (exemplo: atividade sexual insegura, nas sexualmente transmissíveis).

As ConDSS selecionadas foram categorizadas nas dimensões de vulnerabilidade social, individual ou programática, a partir de sua aderência com a definição de cada dimensão.

As ConDSS foram submetidas à avaliação por especialistas enfermeiros selecionados por sua experiência superior a seis meses em atenção primária em saúde na assistência à portadores de DM, atuantes na cidade de Curitiba ou região metropolitana. Não houve critérios de exclusão.

O instrumento de coleta de dados foi um questionário construído em plataforma eletrônica, que continha uma seção de identificação do perfil do profissional e um roteiro com 68 ConDSS. Cada enfermeiro pontuou a influência de cada condição para o desenvolvimento do pé diabético, apresentada por uma escala de Likert com 3 divisões: sem influência, fraca influência e forte influência.

A seleção dos participantes adotou o modelo bola de neve. Inicialmente foram incluídos, intencionalmente, 18 profissionais com o perfil estabelecido. A este grupo foi encaminhada mensagem eletrônica apresentando a pesquisa, convidando à participação e solicitando seu envio para profissionais de sua rede de contato, que contemplassem o perfil estabelecido. Com esta abordagem obteve-se um total de 71 respondentes.

Dos 71 enfermeiros, $77 \%$ atuavam há mais de cinco anos na assistência ao diabético; 82\% eram especialistas; $42 \%$ atuavam na assistência e $20 \%$ na gerência; $80 \%$ eram formados com tempo superior a seis anos; $86 \%$ eram do município de Curitiba; $27 \%$ trabalhavam em instituições de ensino e $24 \%$ em hospitais; $42 \%$ pertenciam ao serviço público municipal.

Para análise dos resultados foi utilizado o índice de validade de conteúdo (IVC), que calcula a concordância entre dois ou mais profissionais e estima a validade de um item. Considera como válidos os itens com IVC acima de $80 \%{ }^{(15)}$. Para estabelecer o IVC foram atribuídos escores $(0,1$ e 2$)$ para as opções de resposta e um total de escore máximo (TM) de 142, o que resultou na equação apresentada na Figura 1.

A pesquisa foi aprovada pelo Comitê de Ética em Pesquisa da Pontifícia Universidade Católica do Paraná sob o parecer número 789.051.

\section{$I V C=((\mathrm{NI} \times 0)+($ Fr $\times 1)+($ Fo $\times 2)) \times 100$ \\ TM}

Figura 1 - Fórmula para cálculo do IVC. Curitiba, PR, Brasil, 2015

Legenda: IVC: Índice de Validade de Conteúdo; NI: nenhuma influência; Fr: Fraca influência; Fo: Forte influência; TM: Total de escore máximo.

Fonte: ALEXANDRE; COLUCI, 2011. 


\section{RESULTADOS}

Da análise do relatório final da CNDSS, foram identificados 95 DSS. Destes, 41 foram selecionados por sua possível relação com o desenvolvimento do pé diabético, sendo organizados em 68 ConDSS (Quadro 1).

Cada uma das dimensões de vulnerabilidade foi composta por um grupo de ConDSS, sendo 35 na dimensão social, 18 na individual e 15 na programática. Após a avaliação dos enfermeiros, 20 ConDSS atingiram IVC superior a $80 \%(29,4 \%$ do total), sendo três na categoria social (15\%); nove na individual (45\%); e oito na programática (40\%). A descrição deste resultado está apresentada nos Quadros 2 a 4.

Quadro 1 - Total de determinantes sociais em saúde (DDS) identificados e selecionados, segundo as camadas do modelo Dahlgren e Whitehead, e respectiva distribuição em condições determinantes sociais em saúde (ConDSS). Curitiba, PR, Brasil, 2015

\begin{tabular}{|lccc|}
\hline Camadas do modelo Dahlgren e Whitehead & DSS Identificados & DSS Selecionados & ConDSS \\
Condições socioeconômicas culturais e ambientais gerais & 8 & 4 & 7 \\
Condições de vida e trabalho & 39 & 17 & 27 \\
Redes sociais e comunitárias & 7 & 6 & 6 \\
Estilo de vida dos indivíduos & 14 & 9 & 15 \\
Idade, gênero e fatores hereditários & 27 & 5 & 13 \\
Total & $\mathbf{9 5}$ & $\mathbf{4 1}$ & $\mathbf{6 8}$ \\
\hline
\end{tabular}

Legenda: DSS: Determinantes Sociais em Saúde; ConDSS: Condição determinante social em saúde.

Quadro 2 - Índice de validade de conteúdo (IVC) para as condições determinantes sociais em saúde (ConDSS) relacionadas aos determinantes sociais em saúde (DSS), classificadas vulnerabilidade individual. Curitiba, PR, Brasil, 2015

\begin{tabular}{|llc|}
\hline DSS & ConDSS & IVC \% \\
Desnutrição & Estar desnutrido & $\mathbf{8 2 , 3}$ \\
Obesidade & Estar desidratado & $\mathbf{8 0 , 9}$ \\
Disponibilidade de alimentos & Estar obeso & $\mathbf{9 7 , 1}$ \\
Alimentação inadequada & Estar com baixo peso & 62,6 \\
& Fazer até 3 refeições ao dia & 62,6 \\
& Fazer de 3 a 6 refeições ao dia & 33,8 \\
& Fazer mais de 6 refeições ao dia & 63,3 \\
Dieta rica em gorduras & Ingerir menos de 2 litros de água por dia & 64,7 \\
Dieta rica em alimentos refinados e processados & Não seguir a dieta recomendada pelos profissionais & $\mathbf{9 5 , 7}$ \\
& Adotar dieta rica em carboidratos e açúcares & $\mathbf{9 8 , 5}$ \\
Dieta pobre em frutas legumes e verduras & Adotar dieta rica em sódio & $\mathbf{8 0 , 2}$ \\
Sedentarismo & Adotar dieta pobre em frutas, legumes e verduras & 61,3 \\
& Praticar atividade física até 1 hora por semana & 64 \\
Tabagismo e alcoolismo & Praticar atividade física entre 1 e 4 horas por semana & 50,7 \\
Doenças pré-existentes & Praticar atividade física mais de 4 horas por semana & 38,7 \\
& Fazer uso de nicotina & $\mathbf{9 1 , 5}$ \\
& Ingerir bebida alcoólica & $\mathbf{8 9 , 4}$ \\
\hline
\end{tabular}

Legenda: DSS: Determinantes Sociais em Saúde; ConDSS: Condição determinante social em saúde; IVC: Índice de validade de conteúdo. 
Na dimensão individual (Quadro 2) as condições consideradas influentes foram: estar desnutrido; estar desidratado; estar obeso; não seguir a dieta recomendada pelos profissionais; adotar dieta rica em carboidratos e açúcares; adotar dieta rica em sódio; fazer uso de nicotina; ingerir bebida alcoólica; ser portador de outras doenças crônicas.

Na dimensão programática (Quadro 3) as condições consideradas influentes foram: não possuir rede de esgoto; difícil acesso à unidade de saúde; não receber atendimento humanizado; realizar até uma consulta médica ao ano; demora em conseguir uma consulta médica; inexistência de ações educativas de incentivo à atividade física na unidade de saúde; inexistência de campanhas em relação aos efeitos nocivos do tabaco; inexistência de ações educativas em saúde.

Na dimensão social (Quadro 4), as condições consideradas influentes foram: morar em casa com más condições de higiene; possuir renda familiar inferior a um salário mínimo; não participar das atividades grupais (recreação) da unidade de saúde.

Quadro 3 - Índice de validade de conteúdo (IVC) para as condições determinantes sociais em saúde (ConDSS) relacionadas aos determinantes sociais em saúde (DSS), classificadas vulnerabilidade programática. Curitiba, PR, Brasil, 2015

\begin{tabular}{|c|c|c|}
\hline DSS & ConDSS & IVC \% \\
\hline Abastecimento de água & Não possuir água encanada & 79,5 \\
\hline Esgotamento sanitário & Não possuir rede de esgoto & 80,9 \\
\hline Luz elétrica & Não possuir luz elétrica & 75,3 \\
\hline Unidade de saúde básica ou saúde da família & Difícil acesso à Unidade de Saúde & 92,9 \\
\hline Humanização & Não receber atendimento humanizado & 84,5 \\
\hline \multirow{6}{*}{$\begin{array}{l}\text { Disponibilidade de consultas médicas/ } \\
\text { enfermagem }\end{array}$} & Realizar até 1 consulta médica ao ano & 85,2 \\
\hline & Realizar de 1 a 3 consultas médicas ao ano & 60,5 \\
\hline & Realizar mais de 3 consultas médicas ao ano & 54,2 \\
\hline & Realizar até 1 consulta com enfermeiro ao ano & 79,5 \\
\hline & Realizar de 1 a 3 consultas com enfermeiro ao ano & 61,2 \\
\hline & Realizar mais de 3 consultas com enfermeiro ao ano & 45,7 \\
\hline $\begin{array}{l}\text { Disponibilidade de prestadores de serviço } \\
\text { especializado }\end{array}$ & Demora em conseguir uma consulta especializada & 90,1 \\
\hline Incentivo à atividade física & $\begin{array}{l}\text { Inexistência de ações educativas de incentivo à } \\
\text { atividade física na unidade de saúde }\end{array}$ & 85,2 \\
\hline $\begin{array}{l}\text { Disponibilidade de campanhas e medidas } \\
\text { adotadas em relação aos efeitos nocivos do } \\
\text { tabaco }\end{array}$ & $\begin{array}{l}\text { Inexistência de campanhas em relação aos efeitos } \\
\text { nocivos do tabaco }\end{array}$ & 84,5 \\
\hline Existência de ações educativas em saúde & Inexistência de ações educativas em saúde & 91,5 \\
\hline
\end{tabular}

Legenda: DSS: Determinantes Sociais em Saúde; ConDSS: Condição determinante social em saúde; IVC: Índice de validade de conteúdo. 
Quadro 4 - Índice de validade de conteúdo (IVC) para as condições determinantes sociais em saúde (ConDSS) relacionadas aos determinantes sociais em saúde (DSS), classificadas vulnerabilidade social. Curitiba, PR, Brasil, 2015

\begin{tabular}{|c|c|c|}
\hline DSS & ConDSS & IVC \% \\
\hline Educação & Ausência de acesso à escola & 77,4 \\
\hline \multirow[t]{2}{*}{ Diferenciais de exposição a riscos } & Morar em zona urbana & 36,6 \\
\hline & Morar em zona rural & 53,5 \\
\hline \multirow[t]{3}{*}{ Moradias insalubres } & Morar em casa pouco iluminada & 62,6 \\
\hline & Morar em casa com presença de umidade & 67,6 \\
\hline & Morar em casa com más condições de higiene & 85,9 \\
\hline Transporte público & Não possuir acesso a transporte público & 62,6 \\
\hline Desemprego & Estar desempregado & 72,5 \\
\hline Trabalho informal & Trabalhar em condições informais & 54,9 \\
\hline Acidentes de trabalho & $\begin{array}{l}\text { Uso de uniforme e/ou equipamentos de proteção } \\
\text { individual inadequado para a função }\end{array}$ & 69,7 \\
\hline Trabalho rural & Trabalhar em ambiente externo & 65,4 \\
\hline Acesso à internet & Não ter acesso à internet & 24,6 \\
\hline Acesso a telefone celular & Não ter acesso a telefone celular & 26 \\
\hline \multirow{4}{*}{$\begin{array}{l}\text { Acesso a tecnologia de informação e } \\
\text { comunicação }\end{array}$} & Não ter acesso a telefone fixo & 28,8 \\
\hline & Não ouvir rádio & 35,9 \\
\hline & Não ler jornais ou revistas & 38,7 \\
\hline & Não assistir televisão & 38 \\
\hline \multirow[t]{4}{*}{ Cor da pele } & Ter cor da pele branca & 33,8 \\
\hline & Ter cor da pele negra & 38,7 \\
\hline & Ter cor da pele parda & 28,1 \\
\hline & Ser índio & 25,3 \\
\hline \multirow[t]{2}{*}{ Gênero } & Ser do gênero feminino & 34,5 \\
\hline & Ser do gênero masculino & 37,3 \\
\hline \multirow[t]{3}{*}{ Renda familiar } & Possuir renda familiar inferior a 1 salário mínimo & 83,1 \\
\hline & Possuir renda familiar entre 1 e 3 salários mínimos & 61,2 \\
\hline & Possuir renda familiar superior a 3 salários mínimos & 38 \\
\hline \multirow[t]{3}{*}{ Escolaridade } & Ter escolaridade inferior a 4 anos & 78,1 \\
\hline & Ter escolaridade entre 4 e 8 anos & 52,1 \\
\hline & Ter escolaridade superior a 8 anos & 44,3 \\
\hline Relações de solidariedade & Não manter contato frequente com os amigos & 52,8 \\
\hline Relações de confiança & Não possuir rede familiar & 71,1 \\
\hline Contato com amigos e parentes & Morar sozinho & 64,7 \\
\hline Pertencimento a grupo religioso & Não frequentar instituições religiosas & 35,2 \\
\hline Pertencimento a associações sindicais & Não pertencer a redes sociais (grupos de convivências) & 45,7 \\
\hline $\begin{array}{l}\text { Pertencimento a atividades de grupos de } \\
\text { recreação }\end{array}$ & $\begin{array}{l}\text { Não participar das atividades grupais (recreação) da } \\
\text { unidade de saúde }\end{array}$ & 80,9 \\
\hline
\end{tabular}

Legenda: DSS: Determinantes Sociais em Saúde; ConDSS: Condição determinante social em saúde; IVC: Índice de validade de conteúdo. 


\section{DISCUSSÃO}

Estudo $^{(16)}$ que analisou a utilização, pela enfermagem, do conceito de vulnerabilidade aponta para o privilégio oferecido à dimensão individual, com discussão superficial dos fenômenos sociais. Este aspecto parece não ter sido superado pelos enfermeiros que atuam na atenção primária, pois o resultado desta pesquisa também privilegia condições relacionadas ao indivíduo e ao biológico, com pouca aproximação às condições sociais.

Para os especialistas consultados no presente estudo, a vulnerabilidade individual é caracterizada, prioritariamente, por condições alimentares, em especial, a dieta rica em carboidratos e consequente obesidade. Ressalta-se que todas as condições apontadas como influentes pelos enfermeiros são fatores de risco evidenciados em literatura(17-20).

O resultado verificado leva a crer que as condições biológicas da dimensão individual são aspectos abordados rotineiramente na avaliação do pé diabético, portanto, importantes para o estabelecimento de vulnerabilidade. Entretanto, para que se visualizem os determinantes sociais, estas condições não deveriam ser analisadas isoladamente, pois se corre o risco de indicar intervenções rotineiras, desconectadas da realidade social do indivíduo portador de DM.

Por outro lado, condições que foram avaliadas como não influentes são consideradas como protetoras e promotoras de saúde individual, dentre elas o número de refeições e de atividade física, bem como a dieta rica em frutas, legumes e verduras.

Estudo apontou que os determinantes sociais relacionam-se diretamente ao controle glicêmico e à redução das complicações, deste modo o foco das intervenções relacionadas ao DM deve ser ampliado, considerando o estilo de vida e as ações de autocuidado realizadas e as que ainda não são internalizadas na rotina diária ${ }^{(21)}$.

Condições que estabelecem vulnerabilidade programática foram relacionadas, prioritariamente, ao acesso à unidade de saúde, à consulta especializada e às ações educativas gerais e específicas. Ao relacionar o conceito de vulnerabilidade aos aspectos que dizem respeito à disponibilidade de recursos destinados à proteção das pessoas, indicar o acesso como um item prioritário pode refletir em uma modificação, superando as intervenções centradas no serviço, direcionando-as para necessidade de saúde do indivíduo ${ }^{(13)}$.

Incluir a ausência de ações educativas como condição de vulnerabilidade programática é um aspecto importante, entendendo-as como promotoras de autocuidado. Pesquisa ${ }^{(22)}$ que identificou o perfil dos diabéticos participantes de um programa de saúde revelou que $22 \%$ dos pesquisados receberam orientações relacionadas aos cuidados com o pé diabético, destes $54 \%$ afirmaram que o responsável pelas orientações foi o enfermeiro.

Artigo que apresenta a análise de uma intervenção educativa baseada na comunicação participativa em relação aos cuidados com os pés dos pacientes portadores de DM, verificou que a mesma trouxe benefícios em relação ao autocuidado, à aprendizagem e à escolha de condutas mais adequadas ${ }^{(23)}$. Deste modo, enfermeiros devem construir práticas educativas que superem as práticas de educação prescritiva.

Estudo que avaliou os cuidados preventivos ao pé diabético no espaço da atenção primária concluiu que estratégias baseadas em tecnologias leve e leve-dura devem ser utilizadas nas avaliações sistemáticas dos diabéticos. Também aponta que a avaliação tem como objetivo sensibilizar o indivíduo para desenvolvimento de habilidades para o autocuidado ${ }^{(24)}$.

Apenas três condições sociais foram indicadas como influentes para o desenvolvimento do pé diabético. Na literatura, a renda familiar é relatada como prevalente no perfil sociodemográfico de portadores de pé diabético ${ }^{(25)}$ e as condições de higiene do portador de DM e sua moradia são relatadas como preditoras de complicações ${ }^{(26-27)}$.

Entretanto a condição social relacionada ao pertencimento a grupos sociais ou a grupos recreativos, na literatura, parece ser associada aos grupos educativos derivados das ações programadas aos 
diabéticos, que envolvem identificação de $\operatorname{ris} \cos ^{(27)}$.

Durante a consulta de enfermagem e consequente avaliação do pé de um diabético, o enfermeiro deve superar o olhar focado ao risco. Uma revisão integrativa sobre o processo de viver humano e a enfermagem sob a perspectiva da vulnerabilidade, conclui que o enfermeiro deve reconhecer diferentes manifestações das vulnerabilidades e refletir sobre as desigualdades para contribuir com o fortalecimento da cidadania e da qualidade dos serviços ${ }^{(28)}$.

É preocupante que condições relacionadas às desigualdades de gênero e etnia, bem como de acesso às tecnologias comunicacionais não tenham sido apontadas como influentes para estabelecer vulnerabilidade social relacionando-as a uma doença crônica.

Estudos apontam para necessidade do reconhecimento sobre a importância dos determinantes sociais para construção e organização das estratégias de cuidado para o enfrentamento e controle do $\mathrm{DM}^{(28-29)}$. À luz dos resultados apresentados pela presente investigação, sugere-se que tal necessidade se incorpora de forma incipiente pelos enfermeiros da atenção básica.

Foram identificadas algumas limitações no presente estudo, dentre elas salientam-se: a ausência de segunda rodada para avaliação de condições muito próximas ao IVC de $80 \%$; a possível influência da organização dos serviços públicos nas cidades de origem dos participantes; o perfil do profissional especialista, pois a realidade do sistema de saúde e local de trabalho refletem diferentes necessidades de saúde dos usuários.

\section{- CONCLUSÃO}

O presente estudo permite afirmar que as ConDSS relacionadas à dimensão social da vulnerabilidade ainda são fracamente reconhecidas pelos enfermeiros como capazes de influenciar no desenvolvimento do pé diabético. A vulnerabilidade individual e programática são as mais relevantes na opinião dos enfermeiros pesquisados, o que coincide com a literatura analisada.

Sabe-se que a avaliação do risco biológico para os portadores de DM é praticada, mas tem sido insuficiente para evitar os agravos do pé diabético. De acordo com os resultados desta pesquisa, podese considerar que a inclusão da avaliação das dimensões de vulnerabilidade associadas à avaliação de risco poderá trazer benefícios na prevenção do desenvolvimento do pé diabético.

Este estudo disponibiliza as ConDSS identificadas e que podem estar relacionadas ao desenvolvimento do pé diabético para estudos futuros.

\section{- REFERÊNCIAS}

1. Sociedade Brasileira de Diabetes. Diretrizes da Sociedade Brasileira de Diabetes: tratamento e acompanhamento do diabetes mellitus. São Paulo: Sociedade Brasileira de Diabetes; 2013.

2. Alvarsson A, Sandgren B , Wendel C, Alvarsson M, Brismar K. A retrospective analysis of amputation rates in diabetic patients: can lower extremity amputations be further prevented. Cardiovasc. Diabetol. [Internet] 2012; 11(18) [acesso em 30 ago 2013]. Disponível: http://dx.doi.org/10.1186/1475-2840-11-18.

3. Haddad MCFL, Bortoletto MSS, Silva RS. Amputação de membros inferiores de portadores de diabetes mellitus: análise dos custos da internação em hospital público. Cienc Cuid Saude. [Internet] 2010; 9(1) [acesso em 30 ago 2013]. Disponível: http://dx.doi.org/10.4025/cienccuidsaude.v9i1.10536.

4. Hobizal KB, Wukich DK. Diabetic foot infections: current concept review. Diabet Foot Ankle. [Internet] 2012; (3) [acesso em 05 set 2013]. Disponível: http://dx.doi.org/10.3402/dfa.v3i0.18409.

5. Ministério da Saúde (BR). DATASUS [Internet] 2010 [acesso em 25 fev 2015]. Disponível: http://www2.datasus. gov.br/DATASUS/index.php. 
6. World Health Organization (WHO). Improving health care: individual interventions. In: World Health Organization. Global status report on noncommunicable diseases 2010. Geneva: World Health Organization; 2011. p.61-71.

7. Bahia. Secretaria de Saúde do Estado da Bahia. Protocolos clínicos para assistência ao diabetes na atenção básica de saúde. Bahia: Secretaria de Saúde do Estado da Bahia; 2014.

8. Ministério da Saúde (BR). Secretaria de Atenção à Saúde. Departamento de Atenção Básica. Estratégias para o cuidado da pessoa com doença crônica: diabetes mellitus. Brasília: Ministério da Saúde; 2013.

9. Londrina. Secretaria Municipal de Saúde. Protocolo clínico de saúde do adulto. Londrina: Secretaria Municipal de Saúde; 2006.

10. Ribeirão Preto. Secretaria Municipal de Saúde. Protocolo de atendimento em hipertensão e diabetes. Ribeirão Preto: Prefeitura Municipal de Ribeirão Preto; 2011.

11. Rio de Janeiro. Secretaria Municipal de Saúde. Superintendencia da Atenção Primária. Guia de referência rápida: diabetes mellitus. Rio de Janeiro: Secretaria Municipal de Saúde; 2013.

12. Ayres JR, Paiva V, França Jr I. From natural history of disease to vulnerability. In: Parker R, Sommer M. Routledge handbook of global public health. London/New York: Routledge; 2010. p. 98-107.

13. Sánchez AIM, Bertolozzi MR. Pode o conceito de vulnerabilidade apoiar a construção do conhecimento em saúde coletiva. Ciênc. saúde coletiva. [Internet] 2007; 12(2) [acesso em 18 mai 2013]. Disponível: http://dx.doi. org/10.1590/S1413-81232007000200007.

14. Sobral A, Freitas CM. Modelo de Organização de indicadores para operacionalização dos determinantes socioambientais da saúde. Saude Soc. [Internet] 2010; 19(1) [acesso em 23 set 2014]. Disponível: http://dx.doi. org/10.1590/S0104-12902010000100004.

15. Alexandre NMC, Coluci MZO. Validade de conteúdo nos processos de construção e adaptação de instrumentos de medidas. Ciênc. saúde coletiva. [Internet] 2011; 16(7) [acesso 30 mar 2014]. Disponível em: http:// dx.doi.org/10.1590/S1413-81232011000800006.

16. Nichiata LYI, Bertolozzi MR, Takahashi RF, Fracolli LA. La utilización del concepto "vulnerabilidad" por enfermería. Rev. Latino-Am Enfermagem. [Internet] 2008; 16(5) [acesso em 15 mai 2014]. Disponível: http://dx.doi. org/10.1590/S0104-11692008000500020.

17. Ahmad W, Khan IA, Ghaffar S, Al-Swailmi FK, Khan I. Risk factors for diabetic foot ulcer. J Ayub Med Coll Abbottabad. [Internet] 2013;25(1-2) [acesso em 14 jan 2014]. Disponível: http://www.ayubmed.edu.pk/JAMC/251/Wasim.pdf.

18. Chellan G, Srikumar S, Varma AK, Mangalanandan TS, Sundaram KR, Jayakumar RV, et al. Foot care practice the key to prevent diabetic foot ulcers in India. The Foot. [Internet] 2012; 22(4) [acesso em 14 jan 2014]. Disponível: http://dx.doi.org/10.1016/j.foot.2012.08.007.

19. Markuson M, Hanson D, Anderson J, Langemo D, Hunter S, Thompson P, et al. The relationship between hemoglobin $\mathrm{A}(1 \mathrm{c})$ values and healing time for lower extremity ulcers in individuals with diabetes. Adv Skin Wound Care. [Internet] 2009; 22(8) [acesso em 14 jan 2015]. Disponível: http://dx.doi.org/10.1097/01. ASW.0000358639.45784.cd.

20. Sarasa-Renedo A, Sordo L, Molist G, Hoyos J, Guitart AM, Barrio G. Principales daños sanitarios y sociales relacionados con el consumo de alcohol. Rev. Esp. Salud Publica. [Internet] 2014; 88(4) [acesso em 14 jan 2015]. Disponível: http://dx.doi.org/10.4321/S1135-57272014000400004.

21. Walker RJ, Gebregziabher M, Martin-Harris B, Egede LE. Relationship between social determinants of health and processes and outcomes in adults with type 2 diabetes: validation of a conceptual framework. BMC Endocr Disord. x[Internet] 2014; 14:82 [acesso em 23 ago 2015]. Disponível: http://dx.doi.org/10.1186/1472-6823-1482002E.

22. Audi EG, Moreira RC, Moreira ACMG, Pinheiro EFC, Mantovani MF, Araújo AG. Avaliação dos pés e classificação do risco para pé diabético: contribuições da enfermagem. Cogitare Enferm. [Internet] 2011; 16(2) [acesso em 16 
dez 2015]. Disponível: http://dx.doi.org/10.5380/ce.v16i2.19975.

23. Rodríguez P, Godoy MC, Mazzo S, Nogueira A, Trevizan PC, Mendes MA, et al. Diabetic foot care before and after an educative intervention. Enfermería Global [Internet] 2013; (29) [acesso em 14 jan 2015]. Disponível: http:// revistas.um.es/eglobal/article/viewFile/154791/143831.

24. Andrade NHS, Sasso-Mendes KD, Faria HTG, Martins TA, Santos MA, Teixeira CRS, et al. Pacientes com diabetes mellitus: cuidados e prevenção do pé diabético em atenção primária à saúde. Rev. enferm. UERJ. [Internet] 2010; 18(4) [acesso em 20 fev 2014]. Disponível: http://www.facenf.uerj.br/v18n4/v18n4a19.pdf.

25. Przysiezny A, Rodrigues KF, Santiago LH, Silva MCV. Características sociodemográficas de pacientes com diabetes mellitus portadores de pé diabético e ou retinopatia diabética atendidos em 16 unidades de estratégia de saúde da família de Blumenau. ACM arq. catarin. med. [Internet] 2013; 42(1) [acesso em 16 jan 2015]. Disponível: http://www.acm.org.br/revista/pdf/artigos/1216.pdf.

26. Fard AS, Esmaelzadeh M, Larijani B. Assessment and treatment of diabetic foot ulcer. Int J Clin Pract. [Internet] 2007; 61(11) [acesso em 14 jan 2015]. Disponível: http://dx.doi.org/10.1111/j.1742-1241.2007.01534.x.

27. Vaquero C. Pie diabético. Valladolid: Andrés Martín; 2012.

28. Barra DCC, Lanzoni GMM, Maliska ICA, Sebold LF, Meirelles BHS. Processo de viver humano e a enfermagem sob a perspectiva da vulnerabilidade. Acta paul. enferm. [Internet] 2010; 23(6) [acesso em 16 jul 2014]. Disponível: http://dx.doi.org/10.1590/S0103-21002010000600018.

29. Newton P, Sasha S, Koula A. Marrying contraditctions: health care professionals perceptions of empowerment in the care of people with type 2 Diabetes. Patient Educ Couns. [Internet] 2011; 85(3) [acesso em 23 out 2014]. Disponível: https://dx.doi.org/10.1016/j.pec.2011.03.015. 